\title{
Lack of Vascular Nitric Oxide Results in Basement Membrane Thickening in Galactose Induced Diabetic Retinopathy
}

\author{
E. Ann Ellis ${ }^{*}$ and Maria B. Grant ${ }^{* *}$
}

*Microscopy and Imaging Center, BSBW 119/MS 2257, Texas A \& M University, College Station, TX 77843 and ${ }^{* *}$ Department of Pharmacology and Therapeutics, P. O. Box 100267, College of Medicine, University of Florida, Gainesville, FL 32610

Diabetes is a disease of vascular complications and capillary basement membrane (CBM) thickening is an ultrastructural hallmark of diabetic retinopathy. In diabetes, hyperglycemia stimulates increased levels of superoxide through the sorbitol pathway. Hyperglycemia stimulates inducible NO (iNOS) which produces NO levels an order of magnitude higher than the levels produced by the constitutive endothelial NOS in the vascular endothelium. Elevated levels of NO and superoxide can uncouple constitutive NOS and reduce availability of NO in the vascular endothelium [1]. Superoxide and NO react to produce peroxynitrite which results in reduced availability of NO. Inhibition of iNOS results in coronary microvascular remodeling in rats [3] and interstitial fibrosis is exacerbated in kidneys of mice lacking the gene for iNOS [4]. Numerous studies have investigated pharmacological inhibition of constitutive and inducible NOS; however, there is question as to the specificity of these drugs and the issue of side effects. Mice with gene deletions of specific NOS isoforms provide a better experimental design for investigating the role of $\mathrm{NO}$ in vascular pathology. We investigated the role of $\mathrm{NO}$ in $\mathrm{CBM}$ thickening in galactose induced diabetic retinopathy [5] in three strains of mice: wild type C57BL/6J (WT), eNOS ${ }^{-1-}$ [eNOS knockout (eNOS KO)], and iNOS $^{-/}[$iNOS knockout (iNOS KO)].

Breeder pairs of WT, eNOS KO, and iNOS KO mice were obtained from Jackson Labs (Bar Harbor, $\mathrm{ME}$ ). Male mice of each strain were fed a rodent diet enriched with 30\% galactose (Purina Mills, Richmond, IN); age matched control mice were fed a normal rodent diet (Purina, St. Louis, MO). Mice were sacrificed after 3 months and 12 months on the galactose diet. Eyes were enucleated, fixed, processed for cytochemical localization of NADH oxidase (marker for superoxide), and embedded in epoxy resin. Nitrotyrosine (marker for peroxynitrite and reduced availability of NO) was localized by colloidal gold based immunocytochemistry on sections where NADH oxidase had been localized [2]. CBM thickness was measured by the orthogonal intercept method [6].

NADH oxidase, the major source of vascular superoxide, levels were elevated in all mice on the galactose enriched diet (Table 1). Nitrotyrosine levels were elevated in the WT and eNOS KO mice on the galactose enriched diet; however, nitrotyrosine levels were not elevated in any of the iNOS KO mice (Table 1). There was increased CBM thickening in the retinas from WT and iNOS KO mice after 12 months on the galactose enriched diet (Table 2). The CBM thickness of eNOS KO mice at 3 months on both the control and galactose diets was 1.8X the CBM thickness of WT and iNOS KO mice at 3 months and comparable to the CBM thicknesss of WT and iNOS KO mice on the galactose enriched diet for 12 months.

Increased CBM thickness in eNOS KO mice without galactosemia indicates a role for NO in vascular homeostasis. The increased CBM thickness in galactose fed WT and iNOS KO mice points to the long term effect of reduced availability of NO in the galactose induced model of diabetic 
retinopathy as a result of removal of NO by reaction with excess superoxide to form peroxynitrite as detected by nitrotyrosine. Lower levels of nitrotyrosine in the iNOS KO mice are a reflection of the lower levels of NO produced by eNOS as compared to the higher levels of NO produced by iNOS in the WT and eNOS KO mice. These data suggest that inhibition of iNOS is not a viable treatment for stopping CBM thickening in diabetic retinopathy and strategies to reduce hyperglycemia (source of superoxide) are more important in long term treatment to prevent diabetic vascular complications.

References

[1] H. Li et al., Nitric Oxide 7 (2002) 149.

[2] E. A. Ellis et al., Nitric Oxide 6 (2002) 295.

[3] K. Numaguchi et al., Hypertension 26(1995) 957.

[4] D. Hochberg et al., Lab. Invest. 80 (2000) 1721.

[5] R. L. Engermann and T. S. Kern, Diabetes 33(1984) 97.

[6] E. B. Jensen et al., J. Microsc. 115 (1979) 19.

[7] This work was supported by NIH/NEI grants EY012601 and EY007739 and JDRF 4-2000-847.

\begin{tabular}{lcc}
\hline & $\begin{array}{c}\text { PERCENTAGE VESSELS } \\
\text { POSITIVE FOR } \\
\text { NADH OXIDASE }\end{array}$ & $\begin{array}{c}\text { NITROTYROSINE } \\
\text { LOCALIZATION } \\
\text { Gold Particles } / \mathbf{5 0} \boldsymbol{~ m}^{\mathbf{2}}\end{array}$ \\
\hline WT Normal Diet & $50.1 \pm 4.7$ & $10.7 \pm 3.8$ \\
\hline WT Galactose Diet & $\mathbf{8 8 . 9} \pm \mathbf{5 . 1}$ & $\mathbf{3 1 . 7} \pm \mathbf{5 . 4}$ \\
\hline iNOS KO Normal Diet & $49.0 \pm 1.4$ & $8.1 \pm 4.0$ \\
\hline iNOS KO Galactose Diet & $\mathbf{7 2 . 0} \pm \mathbf{1 1 . 1}$ & $10.6 \pm 4.3$ \\
\hline eNOS KO Normal Diet & $41.1 \pm 12.7$ & $5.45 \pm 3.7$ \\
\hline eNOS KO Galactose Diet & $\mathbf{8 1 . 9} \pm \mathbf{7 . 2}$ & $\mathbf{3 7 . 9} \pm \mathbf{1 0 . 2}$ \\
\hline
\end{tabular}

Table 1. Localization of NADH oxidase and nitrotyrosine in blood vessels in neural retina of age matched male mice on diets of normal rodent chow and 30\% galactose enriched rodent diet. Numbers in bold type are significantly different, $p=0.05$.

\begin{tabular}{lcc}
\hline & CBM THICKNESS ( nm) & CBM THICKNESS ( nm) \\
& 3 months & 12 months \\
\hline WT Normal Diet & $49.4 \pm 8.9$ & $65.5 \pm 11.0$ \\
\hline WT Galactose Diet & $51.4 \pm 11.3$ & $\mathbf{9 3 . 8} \pm \mathbf{1 4 . 5}$ \\
\hline iNOS KO Normal Diet & $47.6 \pm 9.9$ & $59.9 \pm 10.0$ \\
\hline iNOS KO Galactose Diet & $52.2 \pm 11.6$ & $\mathbf{9 0 . 6} \pm \mathbf{1 3 . 6}$ \\
\hline eNOS KO Normal Diet & $\mathbf{8 5 . 6} \pm \mathbf{1 8 . 9}$ & Mice did not survive. \\
\hline eNOS KO Galactose Diet & $\mathbf{8 9 . 5} \pm \mathbf{1 6 . 1}$ & Mice did not survive. \\
\hline
\end{tabular}

Table 2. CBM thickness in neural retina of age matched male mice on diets of normal rodent chow and $30 \%$ galactose enriched rodent diet. Numbers in bold type are significantly different, $\mathrm{p}=0.05$. 